Slavica

bruxellensia

\section{Slavica bruxellensia}

Revue polyphonique de littérature, culture et histoire

slaves

$3 \mid 2009$

Pensée des hommes

\title{
Ruženje naroda u dva dela /Outrage au peuple en deux parties (Extraits traduits du serbo-croate par Nataša Džigurski-Dubajić)
}

\section{Nataša Džigurski-Dubajić}

\section{(2) OpenEdition}

\section{Journals}

\section{Édition électronique}

URL : http://journals.openedition.org/slavica/205

DOI : $10.4000 /$ slavica. 205

ISSN : 2034-6395

\section{Éditeur}

Université libre de Bruxelles - ULB

Édition imprimée

Pagination : 87-97

ISSN : 2031-7654

\section{Référence électronique}

Nataša Džigurski-Dubajić, « Ruženje naroda u dva dela /Outrage au peuple en deux parties (Extraits traduits du serbo-croate par Nataša Džigurski-Dubajić) », Slavica bruxellensia [En ligne], 3 | 2009, mis en ligne le 01 avril 2011, consulté le 21 avril 2019. URL : http://journals.openedition.org/slavica/205 DOI : 10.4000/slavica.205

Ce document a été généré automatiquement le 21 avril 2019

\section{(c) (1) (3)}

Les contenus de Slavica bruxellensia sont mis à disposition selon les termes de la Licence Creative Commons Attribution - Pas d'Utilisation Commerciale - Pas de Modification 3.0 France. 


\title{
Ruženje naroda u dva dela /Outrage au peuple en deux parties (Extraits traduits du serbo-croate par Nataša Džigurski-Dubajić)
}

\author{
Nataša Džigurski-Dubajić
}

\section{NOTE DE L'ÉDITEUR}

Toutes les notes sont de la traductrice

\section{Présentation}

Lorsqu'en 1968 Slobodan Selenić (1933-1995) publie son roman, Memoari Pere Bogalja (Mémoires de Pera l'infirme), il est déjà connu en tant que critique de théâtre et journaliste. Dans ce premier roman, Selenić annonce ses grands thèmes, ses obsessions que l'on retrouvera dans les œuvres qui suivront. Comme tout écrivain « obsessionnel », il ne changera jamais de registre. Sa plume apportera à la littérature serbe des romans poignants du réalisme critique, une triste saga sur le déclin de la bourgeoisie sous le poids de la révolution bolchevique.

Dans tous les romans de Selenić, les circonstances historiques ont une influence déterminante sur le caractère des idées, sur le développement de l'action et sur le destin des personnages car ils sont tous, sans exception, réalisés en tant qu'objets avec lesquels joue l'Histoire, de simples objets passifs nécessaires à la construction de ce refrain sur l'incapacité de l'homme à agir contre l'invasion d'idéologies désolatrices. Selenić est un écrivain qui possède une conscience critique historique aiguë mais aussi une profonde compréhension du caractère dramatique des changements de la morale au sein d'une société chamboulée par un enchaînement de faits historiques. D'une oeuvre à l'autre, il 
approfondit la question cruciale de l'intégrité morale de l'homme moderne sur les décombres de l'Histoire. En d'autres termes, l'auteur dépeint un drame de conscience de la civilisation actuelle, une conscience chargée d'actes contradictoires par un abandon trop facile à la violence aveugle qui s'est libérée au cours de ce dernier siècle.

Le monde qu'il décrit est né de la collision entre la bourgeoisie serbe de l'entre-deuxguerres, qui n'a pas eu le temps de prendre racine, et d'une nouvelle classe, faite de révolutionnaires montagnards qui subira pourtant des transformations en acceptant les bienfaits et les privilèges accordés aux vainqueurs et deviendra en quelques années ce que l'on appelle la «bourgeoisie rouge ». C'est de ce contact entre deux cultures, deux civilisations, deux mentalités incompatibles que découlent pratiquement tous les thèmes obsessionnels de Selenić.

C'est l'Histoire qui détermine le destin des personnages de Selenić, toujours tragique. La vérité objective est selon toute apparence une notion qui préoccupe l'auteur. Il n'a jamais prôné une vérité unique. C'est pour cela que dans ses romans, il confronte des vérités individuelles. Le roman est, d'après l'auteur, le moyen parfait de donner forme à un système d'idées à travers des personnages concrets.

Ruženje naroda $u$ dva dela (Outrage au peuple en deux parties) est une pièce de théâtre et par conséquent une forme d'expression brève. Néanmoins, elle présente l'avantage de mettre en scène pratiquement tous les questionnements de Selenić. Il s'agit d'une sorte de synthèse de toute sa poétique.

La piècea été écrite à une époque durant laquelle elle ne pouvait que se heurter au rejet. En cette fin des années 1980, les nationalismes, sommeillant depuis longtemps, atteignaient leur apogée avant de se libérer dans la violence lors de la dernière décennie du XXe siècle.

\section{Traduction}

VI (Partie 1)

Cellule numéro sept. Tous les prisonniers sont réunis

Jezdimir :

Tu dis, Stevan, le 22 novembre ${ }^{1}$ ? À Florence ? Je ne connaissais pas cette date jusqu'à ce jour. Puis le $1^{\text {er }}$ décembre ${ }^{2}$ Un grand jour, terrible, pour les Serbes. Puis le 20 juin ${ }^{3} . . . E h$, si seulement on avait d'autres Puniša

Stevan :

Et si seulement le 29 décembre ${ }^{4}$ avait été accueilli comme il se doit.

Obrad:

Puis le 9 octobre ${ }^{5} .$.

Jezdimir :

Comment sais-tu, toi, pour le 9 octobre, puisque tu n'étais même pas né alors... 
Obrad:

J'étais né. Je suis venu au monde et j'ai pleuré à chaudes larmes avec le peuple.

Stevan :

Puis la catastrophe, le 26 août ${ }^{6}$ !

Jezdimir :

Puis la deuxième catastrophe, le 17 avrill $^{7}$ ! Mais vient l'aube du 10 mai $^{8} 1941$ !

Stevan:

Puis le 19 septembre à Struganik ${ }^{9}$, puis le 26 octobre à Brajićn ${ }^{10}$, finalement, le 2 novembre ${ }^{11}$ près de Užice - le torchon brûle!

Jezdimir :

Ensuite le 12 septembre ${ }^{12}$ noir du roi, espèce de mollasson au cul malpropre !

Le pope Sava :

Ne dites pas ça, mes enfants, il est comme il est, c'est notre roi tout de même !

Stevan:

Dites donc, le 7 août ${ }^{13}$ à Rome a été pire. De la ville papale, jamais les bonnes nouvelles ne nous parvenaient, à nous autres Serbes !

Čapajev :

Hé, n'est-ce pas le jour où le camarade Maréchal a rencontré Alexander?

(Ils ne répondent pas, ils rient)

Pour sûr qu'il a été noir pour vous !

Stevan:

Ça a été démontré, c'était clair comme de l'eau de roche : les plus grands ennemis des Serbes sont les Anglais.

Le pope Sava :

C'est exact, mon fils, ils ne se soucient pas du Serbe, ils nous ont laissé sur le sable, mais ce ne sont pas les pires. Pour un Serbe, les pires tyrans, sans Dieu ni foi, ce sont les communistes russes.

Obrad:

Et les Turcs?

Jezdimir :

On se fout des Turcs! Leur temps est révolu.

Obrad:

Tu parles! Et nos Turcs $^{14}$ à nous ?

Jezdimir :

Eux, je ne dis pas, ce sont des tyrans. Ils nous détestent tout autant.

Stevan:

Et vous, qu'en dites-vous, Slavoljub? 


\section{Slavoljub :}

Je ne sais pas. Personne ne mentionne les Magyars ${ }^{15}$, alors qu'ils en ont égorgé tant des nôtres partout en Bačka ${ }^{16}$. Ici, en Serbie, ça ne compte pas. Nous n'avons de cesse de ressasser nos propres griefs, mais c'est peut-être le Magyar qui méprise le plus le Serbe.

Stevan :

Il le méprise peut-être, mais il ne le déteste pas autant que ne le fait l'Albanais.

Obrad :

Le Bulgare nous déteste encore plus.

Čapajev :

Bande de connards de tchetniks, et les Allemands, les Italiens, ils ne vous viennent pas à l'esprit !?

Stevan :

Ça, ça va de soi. Mais calcule bien, Čapajev, combien de Serbes ont été tués par les Allemands et les Italiens réunis, et puis regarde les Croates, combien en ont-ils massacrés. C'est le Croate, le pire ennemi des Serbes.

Jezdimir :

Qu'ils aillent au diable ! Comme ennemi, suppôt de Satan, il n'y en a pas de pire. Sais-tu ce qu'il dit ce Croate, cette espèce de prêtre défroqué, celui qui a été curé catholique et qui a fini tchetnik...?

Le pope Sava :

Vilović.

\section{Jezdimir :}

Voilà, lui. Il dit: Les Croates n'existent même pas. Ceux qui utilisent le dialecte kajkavien ${ }^{17}$, ce sont les Slovènes ; ceux qui utilisent le štokavien ${ }^{18}$ sont des Serbes venus de Bosnie en Dalmatie. Ce n'est même pas un peuple, qu'il aille se faire foutre, celui qui les a engendrés et qui les a dressés contre nous !

Stevan :

Attends, voïvode, s'ils n'existent donc pas, qui nous égorgeait alors?

Čapajev :

(il rit)

Les Serbes štokaviens et les Slovènes kajkaviens !

Jezdimir :

Qu'est-ce que t'as à glousser comme ça, abruti ! De toute façon, ce sont des renégats.

Obrad:

Non mais, toutes les conditions ont été réunies pour anéantir les Serbes jusqu'au dernier! Pour sûr, le Serbe est venu au monde pour être la tête de Turc de tous.

Jezdimir :

Allez, Obrad. Le chapelet serbe. Donne-moi dans l'ordre toutes les villes, sans oublier le moindre petit lieu.

Obrad:

Je commence à partir de Split ou de la Slovénie? 
Jezdimir :

Vas-y, de la Slovénie.

Obrad:

Banja Loka, Srpske Moravice...

Jezdimir :

Srpske Moravice, l'étoile de mer...

Obrad:

Vojnić, Vrgin most, Dubica...

Jezdimir :

Vrgin mos, Srpska kos.

Obrad:

Novska, Pakrac, Gradiška, Srbac...

Jezdimir :

Serbe, Srbac, du serbe, les Serbes...

Obrad:

Prnjavor, Popovići, Gračanica...

Jezdimir :

Gračanica, à l'image des Serbes, ton prochain est de la ville d'Užice...

Obrad:

Čelić, Koraj, Zagoni...

Jezdimir :

Et, voilà...

Obrad:

Sremska Rača, Irig, Ruma...

Jezdimir :

... Tout en traversant le territoire du peuple serbe, tu es arrivé, mon Obrad, de Slovénie jusqu'à la fière ville de Šabac! Le chapelet serbe, mon cœur s'en réjouit, nos héros serbes! Allez, Stevan, je te le demande comme à un frère, déclame ton Shakespeare. Pas un mot, vous autres! Solennellement! Je ne veux pas entendre une seule mouche voler!

Stevan :

«You blocks, you stones, you worse than senseless things!

O you hard hearts, you cruel men of Rome,

Knew you not Pompey? Many a time and oft

Have you climb'd up to walls and battlements,

To towers and windows, yea, to chimney-tops,

Your infants in your arms, and there have sat

The livelong day, with patient expectation,

To see great Pompey pass the streets of Rome... $»^{19}$ 


\section{VIII (Partie 1)}

Miloš dans le divan, à Kragujevac, en juin 1817. Arrive Vujica Vulićević.

Vujica :

Monseigneur, une missive de Karageorges.

Miloš :

Que dit-il?

Vujica :

Il te salue, il invoque votre parenté.

Miloš :

Qu'est-ce que tu as à tourner autour du pot, là ? Passe !

Vujica :

Il est en colère, qu'il dit, car tu as assassiné ses voïvodes.

Miloš :

Les voïvodes ! De la fiente, pas des voïvodes !

Vujica :

Le Voïvode $\operatorname{Moler}^{20}$...

Miloš :

Le bouffon autrichien, dilapidateur de l'argent du peuple! Et puis, ce n'est pas moi qui l'ai tué, c'est le vizir qui l'a étranglé.

Vujica :

Le Voïvode Pavle Cukić...

Miloš :

Cet agitateur et ce mamelouk! Eh, ma foi, il a perdu la vue à force de reluquer le con. Que veux-tu que je fasse d'un voïvode bigleux?

Vujica :

Le Capitaine Radič Petrović ${ }^{21}$...

Miloš :

(d'un ton ironique)

Dieu l'a voulu. Ainsi, j'ai fait de lui un martyr !

Vujica :

L'Evêque Milentije Nikšić...

Miloš :

Ce n'est pas moi qui l'ai tué, Vujica, mais toi, enfant de catin!

Vujica :

Il est surtout en colère, qu'il dit, car tu as tué le prince Sima Marković !

Miloš :

Ce mollasson de Sima! Il a soulevé une armée contre moi, fichtre! Moi, je tuais pour épargner au peuple une plus grande tuerie, pour obliger ces petits péteux à faire la paix 
avec les Turcs ! Et Karageorges, maudit soit-il, il vaudrait autant avoir affaire à un Turc. Il a tué son propre père d'une balle dans le front, à Crvena Jaruga! Passons. Que dit-il d'autre?

Vujica :

Il dit qu'il a l'intention de venir en Serbie et, avec ton aide, de fomenter une nouvelle insurrection contre les Turcs.

Miloš bondit du divan. D'un signe de main, il ordonne à Vujica de se taire. Il arpente la pièce, perdu dans ses pensées.

Miloš :

Et des Russes? Que dit-il ? Le Tsar va-t-il entrer en guerre contre la Turquie à ce moment-là?

Vujica :

Pas tout de suite, qu'il dit, mais Philiki Hétaïrie ${ }^{22}$ nous épaulera. En même temps que nous, ici, les Grecs se soulèveront en Hellade ${ }^{23}$.

Miloš :

Mon cul! Les Grecs! Nous ne pouvons pas battre la Turquie sans l'aide de la Russie. Nous pouvons seulement tous périr inutilement, comme jadis, en 1813, sous leurs ordres. Dis-lui qu'il reste où il est. Et moi, ma foi, comme je l'ai toujours fait, je monnaierai, pour le peuple, la paix avec les Turcs, avec Marachli Ali-pacha et Istanbul.

Vujica :

Il dit que c'est une honte.

Miloš :

Qu'est-ce qui est honteux?

Vujica :

Eh bien, tout ça, avec les Turcs, les tyrans. Et il est honteux de porter le turban, comme tu le fais.

Miloš :

(Il arrache la lettre des mains de Vujica et la déchire)

Qu'il aille au diable, comment ose-t-il me parler de la sorte! Il voudrait que les Turcs égorgent nos enfants et nous exterminent jusqu'au dernier? Cent têtes serbes orthodoxes contre une seule tête turque! Comment a-t-il pu si vite oublier ce qui s'est passé en 1813, que les vautours lui arrachent le cœur! Une fois de plus, et il ne restera ni Serbes ni Serbie. Et pour quelle satanée raison? Pour que Karageorges gouverne la Serbie! Le peuple ou Karageorges, qu'en dis-tu, Vujica, qui alors vaut-il mieux sacrifier? Devons-nous tous périr ou lui seul?

(Il se calme brusquement, d'une voix basse)

Sais-tu, Vujica Vulićević, qui est mon pire ennemi?

Vujica :

Je le sais, monseigneur.

Miloš :

Dis-le. 
Vujica :

Lui.

Miloš :

C'est ça, Vujica. Ce n'est pas le Turc qui m'inquiète, Vujica. Avec Karageorges, la cause de mes tourments, je ne sais que faire, Vujica.

\section{XIX (Partie 2)}

Stevan et Slavoljub font la corvée de tinette. Ils prennent une tinette en dehors de la scène, renversent son contenu dans un coin et la rapportent vide. A voir leur visage grimaçant de dégoût, nous comprenons que ça sent mauvais, mais ils n'en parlent pas.

\section{Stevan :}

Friends, Romans, countrymen, lend me your ears $!^{24}$

Slavoljub :

Vous en connaissez déjà la moitié?

Stevan:

Eh bien, oui, certains passages. Comme Uča connaît le « soulèvement contre les Dahis ».

Slavoljub :

Pourquoi précisément Jules César?

Stevan :

Eh bien, j'apprécie cette pièce, mais le choix est arbitraire. J'aurais pu commencer à apprendre par cœur n'importe quelle autre pièce. Il faut s'évader. On devient follement avide de solitude en compagnie de gens que l'on ne supporte pas.

Slavoljub :

Qu'est-ce qui vous a incité à vous y mettre?

Stevan :

C'était par hasard. Ça m'amusait de voir le voïvode, qui meuglerait s'il ne parlait pas serbe, pieusement m'écouter réciter. N’auriez-vous pas quelque mégot?

Slavoljub:

Je n'en ai pas, Stevan, je vous ai tout donné.

Stevan :

Comment avez-vous pu abandonner le tabac ? Ici ! Dans le neuvième cercle de l'enfer ?

Slavoljub

Cela m'est plus facile que de compter des moitiés de cigarettes.

Stevan :

Je dois vous faire part de quelque chose, Slavoljub. Je suis content de vous avoir ici. C'est idiot de dire cela à quelqu'un qui est en prison, mais moi, à côté de tous ces 
tchetniks, avec lesquels j'ai fait la guérilla pendant tant d'années, il n'y a qu'avec vous que je trouve des sujets de conversation.

Slavoljub:

Pourquoi vous êtes-vous joint à eux ?

Stevan :

Cela semble être une question logique aujourd'hui. Je faisais alors quelque chose d'inévitable. Pour ne pas perdre toute ma dignité, voilà pourquoi je les ai rejoints. C'est exactement cela, oui. Le pays est occupé, il nous faut le libérer. L'idée de rejoindre les partisans ne m'a, bien entendu, même pas effleuré l'esprit ; en revanche, Draža avait toute la légitimité : soutenu par notre gouvernement légal, les alliés, la Radio de Londres, le peuple qui a fait de lui une légende en un rien de temps. Admettez-le, comment aurait pu agir autrement un patriote, un homme de conscience, que de rejoindre la guérilla, en cette année 1941 ? J'ai été pris de peur, j'avais conscience des expiations qui inévitablement m'attendaient, mais je n'ai pas pu faire autrement.

Slavoljub:

Et lorsque les tchetniks ont ôté au peuple le goût du pain, lorsqu'ils sont devenus pour lui des égorgeurs, des pilleurs...

Slavoljub :

Mais pas Draža. Ses commandants, ses voïvodes, certes ; mais Draža, lui, il a joui jusqu'à la fin d'une notoriété au sein du peuple. D'une juste notoriété, je crois. Il était sincèrement convaincu que le plus important était de sauver le peuple de la chimère communiste. Cela ne veut point dire qu'il n'est pas coupable de l'anéantissement du mouvement tchetnik de Ravna Gora. Étant lui-même un cul-terreux d'Ivanjica, il a laissé la paysannerie engloutir le mouvement. Et la bête paysanne a rompu les barreaux.

Slavoljub:

C'est donc la paysannerie qui est à l'origine de tout?

Stevan :

Pas tout à fait. Ce sont les Anglais qui nous ont trahis. Dragiša Vasić promettait à l'époque qu'une fois la guerre finie, il fonderait à l'université de Belgrade le département de la haine envers les Anglais. Il n'a pas eu le temps de le faire, pauvre bougre. Les Croates l'ont assassiné. En revanche, bien avant que les Anglais ne nous trahissent, j'ai été envahi par la crainte que les partisans ne s'avèrent être plus forts que nous. La faute à ces barreaux! Votre geste de dépit n'a pas lieu d'être. C'est bien à cause d'eux! Entendez cela; nous avons levé, avec les partisans, une assemblée de mobilisation à Mionica. À cette époque, nous étions encore ensemble. Après l'assemblée, le commissaire Dudić, assis à une table, inscrit ceux qui voulaient rejoindre les partisans, et à une autre table, le capitaine Lečić inscrit ceux qui voulaient rejoindre les tchetniks. Arrive alors un paysan d'un certain âge qui dit: «Je voudrais m'inscrire chez les uns et chez les autres ». «Comment ça? », qu'ils lui demandent, et lui : « Pour les partisans à Paune, j'enverrai des chaussettes et des souliers, et pour les tchetniks à Ravna Gora, j'enverrai des brebis et de l'argent. » Après avoir ri un instant, le capitaine Lečić dit : « Ça me va comme ça! »; et Dudić fit sèchement : « Nous ne voulons ni de toi, ni de tes souliers ! Va-t'en chez eux !» 
Slavoljub :

Vous voulez dire que les partisans ont gagné grâce au caractère exclusif du mouvement?

Stevan:

Ils ont gagné parce qu'ils ont su garder derrière les barreaux notre pègre paysanne : la poigne de fer de l'élite dirigeante communiste ou les barreaux horizontaux; la promesse faite à cette bande de voyous qu'elle atteindrait le paradis sans classes ou les barreaux verticaux. Nos animosités étaient parfois vengeresses, mais souvent pillardes, à des fins personnelles. Les leurs, par définition, à quelques nuances près, avaient un caractère religieux, fanatique. Aussi cruels soient-ils, les crimes fanatiques se justifient par une prétendue cause supérieure. Et c'est par le biais de cette cause supérieure que les communistes ont brisé la raison de la foule. Dans le mouvement tchetnik, c'est la foule qui a eu raison de nous. Et croyez-moi, on comptait parmi nous des hommes enthousiastes, purs, intelligents. Au début nous étions nombreux, puis de moins en moins. À la fin, nous n'avions plus de raison d'être. Un tchetnik, c'est ce paysan serbe qui a brisé lesdits barreaux, plein de haine envers la ville, la bourgeoisie, la culture, et qui fait fi de toute valeur sacrée dans une époque de violences irrépréhensibles. Il l'a emporté sur nous. La foule ignorante nous a engloutis, c'est ce que je veux dire. Je me suis mis à haïr, Slavoljub, non pas les tchetniks, non pas les partisans, mais le peuple. Les Serbes. À dire vrai, le peuple ne me supporte pas non plus. Il flaire tout de suite que je ne suis pas un des leurs.

Slavoljub:

Qu'était votre grand-père?

Stevan:

Le peuple.

Slavoljub :

Et votre père, votre mère, vos oncles paternels et maternels ?

Stevan :

Tous, jusqu'au dernier, des paysans.

Slavoljub :

Le peuple, Stevan, ce n'est pas un tramway dans lequel l'on monte ou l'on descend selon nos désirs. Que vous le vouliez ou non, vous aussi, vous êtes partie intégrante de ce peuple. C'est orgueilleux et inexact, ce que vous dites. Notre peuple, tout furieux et tout déchaîné qu'il est, a, pour être tout à fait honnête, fait montre dans cette guerre d'une énergie fratricide inouïe, mais aussi de patriotisme, Stevan, d'endurance. Votre rage à l'égard du peuple est superficielle et imprégnée de snobisme. Ce même peuple, que vous invectivez tant, a tout de même réussi, en l'espace d'une petite centaine d'années, à sortir de l'obscurité orientale épaisse et à entrer en Europe.

Stevan:

Avec tout le respect que je vous dois, la question de l'Europe n'a pas lieu d'être soulevée. Ce même peuple, le vôtre et le mien, ce peuple sauvage, aux mœurs orientales, n'est en Europe que géographiquement. Il est prêt à subir toute humiliation, puis il devient irrespectueux, assoiffé de sang dès qu'il met le grappin sur la moindre parcelle de pouvoir. Le pouvoir, dans la vision serbe, consiste en effet à avoir le droit d'empaler son voisin, sans raison aucune, juste pour que l'on sache qui a le pouvoir. 
Regardez le voïvode! Tuer tout ce qu'il y a de lettré à Toplica! Afrikaner! Regardez Čapajev! Il coupe les oreilles, il pille, il fait arrêter d'innocents maris pour pouvoir chevaucher leurs femmes puis, finalement, il se fait arrêter parce qu'il traitait la direction de tous les noms sous l'emprise de l'alcool. Voyez-vous à quel point lui et le voïvode se ressemblent? Et pas seulement parce qu'ils viennent de villages voisins ! Pourquoi donc nous plaignons-nous? Ces sauvages, qu'ils soient tchetniks ou partisans, représentent quelque chose qui nous revient.

Slavoljub:

Fâchez-vous tant qu'il vous plaira, mais je dois vous le demander : n'êtes-vous pas en train de justifier une certaine...

Stevan:

... déchéance. Ne vous gênez pas...

Slavoljub :

... disons, une certaine nonchalance morale, par l'inexistence d'un ordre moral au sein du peuple?

Stevan :

Slavoljub, ne me faites pas rire. Qu'est-ce que j'aurais à y gagner à me justifier, pour l'amour de Dieu !? Je vis, moi, d'un mégot à l'autre dont on me fait l'aumône. Ce que j'ai pu vous dire ne me concerne pas personnellement. Je n'existe pas, mais, hélas, je le sais : Des pratiques vertueuses se créent sur la durée, car elles n'ont de pire ennemi que la précipitation. À nous autres Serbes, c'est justement le temps qui nous manquait pour consolider les choses. Pour qu'on les garde en mémoire. Pour que l'on s'y consacre.

Slavoljub:

Vous ne pouvez pas vivre avec tant de noirceur en vous.

Stevan :

De noirceur? Ce n'est pas de la noirceur. Comment dirais-je...

Slavoljub:

Tant de haine?

Stevan :

Tant de répugnance. Oui. De répugnance envers le monde.

Slavoljub:

Il y a pire. La répugnance envers soi-même.

Stevan :

Vous vous démenez en vain, Slavoljub. Ne voyez-vous donc pas avec quelle rapidité on s'habitue au pire ? La réalité, c'est cette tinette.

Ils vident les tinettes. 
Miloš et Vujica dans le divan.

Vujica :

Il est chez moi, monseigneur.

Miloš :

Aferim $^{25}$ ! Il veut une insurrection?

Vujica :

En effet. Il dit que la Russie apportera son aide. Ainsi que Philiki Hétaïrie en Hellade.

Miloš :

Qu'il aille se faire foutre, tant que je ne vois pas l'armée russe de mes propres yeux, je n'y crois pas. Ne nous ont-ils pas trahis plus d'une fois? Le Tsar russe se soucie du peuple russe, et du mien, il ne s'en soucie que lorsqu'il en a besoin. Si moi, je ne me soucie pas des Serbes, personne ne le fera. Le peuple ou Karageorges, qu'en dis-tu Vujica, qui alors vaut-il mieux sacrifier? Devons-nous tous périr ou lui seul ? C'est toi Vujica, et comment ! C'est toi qui vas le tuer ! Trancher la tête, et comment ! L'offrir aux Turcs, et comment!

Vujica :

Epargne-moi ça, monseigneur, lui, je ne peux pas.

Miloš :

Si tu veux tant mieux. Si tu ne veux pas, tu n'auras pas le choix.

Vujica :

Voici ce que je pense, monseigneur! Il est fini, notre gouverneur turc. Comme si je ne voyais pas dans quel état est son fief de Belgrade, à lui, le pacha à trois queues ? Ses fenêtres n'ont pas de carreaux, elles sont attachées avec des cordes afin qu'elles ne tombent pas. Des amas d'ordures empêchent l'accès au portail. Des chiens exorbités fouillent les ordures ; son armée est en haillons, les pieds nus, couchée dans la poussière au soleil à toute heure du jour. Il ne pourrait pas l'entretenir toute délabrée qu'elle est, si tu ne lui donnais pas d'argent. C'est lui qui dépend de toi, monseigneur, et pas toi de lui. Alors pourquoi lui offrirais-tu jusqu'à la tête de Karageorges?

Miloš :

Voilà ! Pour ça! Tu penses juste, Vujica, mon voïvode ! Parce que le pacha dépend de moi ! Pierre par pierre, je comblerai le fossé, tout en monnayant les faveurs des Turcs, doucement, doucement, je libérerai la Serbie, et c'est moi seul qui pourrai le faire! Attendons que la Turquie décline encore un peu! Lorsque le glas fatidique sonnera! Lorsque les armées russes et autrichiennes chargeront! Miloš, il sait ce qu'il fait, tu entends !? Et si Karageorges fomente une insurrection, est-ce que Marachli Ali-pacha avec ses chiens et son armée somnolente restera seul ici ? Parle, diable, restera-t-il seul?

Vujica :

Non. Le sultan enverra l'armée.

Miloš :

(il se lève et fait de lentes allées et venues devant Vujica)

C'est toi, Vujica, qui va le tuer... 
Vujica :

Même si tu n'as ni âme...

Miloš :

... lui trancher la tête ...

Vujica :

... ni foi...

Miloš :

... me l'apporter...

Vujica :

... ni Dieu...

Miloš :...

et moi, je l'enverrai au sultan comme présent !

Vujica :

... tu ne dois pas manquer de bon sens. Monseigneur, t'ai-je déjà mal conseillé? Écoute-moi, puis tu feras comme il te plaira : le peuple te tuera.

Miloš :

Doucement, Vujica, ne mets pas la charrue avant les bœufs, maudits soient tes aïeux. Les poiriers seront décorés des cadavres de rebelles, comme le sont les étals des boucheries par les carcasses de mouton.

Vujica :

Je sais que tu es capable de le faire. Mais, ça ne changera rien. J'ai une autre idée.

Miloš :

Parle.

Vujica :

Lorsque la tête partira pour la ville d'Istanbul, notre seul secours sera celui-ci : que mes hommes de main laissent courir la rumeur que tu caches un Karageorges sain et sauf et que la tête envoyée aux Turcs n'est pas la bonne.

Miloš :

Est-ce que le peuple le croira?

Vujica :

Il le croira, il te connait.

Miloš :

(il cesse de marcher, réfléchit)

L'ordure! Tu es pire que moi.

(Il le dit en compliment. Il réfléchit de nouveau, affiche un sourire.)

$\mathrm{Tu}$ es vraiment une belle ordure. Mais, le premier a la primauté, comme disent les Turcs. Va chercher la tête du chef, Vujica, ma brave petite ordure! 


\section{Fuvres de Slobodan Selenić traduites en français :}

2 - L'ombre des aïeux (Očevi i oci, traduit du serbo-croate par Gojko Lukić et Gabriel Iaculli), Gallimard, coll. « Du monde entier », Paris, 1999, 416 p.

- Meurtre avec préméditation, (Ubistvo s predumišljajem, traduit du serbo-croate par Gojko Lukić et Gabriel Iaculli), Gallimard, coll. «Du monde entier », Paris, 1996, 240 p.

- Ces deux hommes (Prijatelji, trauit du serbo-croate par Mireille Robin), Robert Laffont, Paris, 1990, $304 \mathrm{p}$.

\section{NOTES}

1. Malgré tout le soin que nous avons apporté à essayer de trouver ce qui s'est passé à Florence un 22 novembre, nous n'y sommes pas parvenus.

2. $1^{\text {er }}$ décembre 1918 : proclamation de l'union de la Serbie et « des pays de l'État indépendant des Slovènes, Croates, et Serbes dans un royaume uni » par le prince Aleksandar, régent de Serbie.

3. 20 juin 1928 : en pleine assemblée, un député monténégrin, Puniša Račić, fait feu sur les députés du parti paysan croate. Deux meurent sur le coup, Stjepan Radić succombe à ses blessures un mois plus tard. Le parti paysan est désormais dirigé par Vladimir Maček.

4. 1932 : publication du «manifeste de Zagreb» signé par des politiques croates mais aussi des Serbes de Bosnie. Ils réclament la souveraineté populaire et le respect des droits nationaux des Serbes, des Croates et des Slovènes. S'ensuit l'internement des signataires les plus en vue, dont le Croate Maček, condamné à trois ans de prison pour trahison le 24 avril 1933.

5. 9 octobre 1934: attentat de Marseille. Aleksandar I tombe sous les balles d'un terroriste macédonien. Un conseil de régence présidé par le prince Paul exerce le pouvoir au nom du jeune roi Pierre II.

6. Négociations secrètes entre le prince Paul et Maček. Elles aboutissent à l'accord du 26 août 1939: création d'une province de Croatie comptant quatre millions d'habitants, dont 866000 Serbes, avec à sa tête un gouverneur pourvu de pouvoirs étendus et paré du titre, hérité d'une histoire pluriséculaire, de ban de Croatie. Un Sabor (Parlement) doté de compétences régionales est également institué. En d'autres termes, la Croatie accède à l'autonomie tandis que Maček devient vice-Premier ministre de l'État yougoslave.

7. 17 avril 1941 : la Yougoslavie se rend à l'Allemagne.

8. 10 mai 1941 : Draža Mihajlović, le chef des tchetniks, établit l'état-major de la résistance à Ravna Gora.

9. 19 septembre 1941: première rencontre en vue d'une collaboration entre Tito et Draža Mihajlović.

10. 26 octobre 1941 : deuxième rencontre entre Tito et Mihajlović. Celui-ci réussit à obtenir 500 fusils pour ses tchetniks.

11. 2 novembre 1941 : les tchetniks attaquent la ville d'Užice où se trouve le QG de la résistance de Tito et qui est également le centre de la «République d'Užice», le territoire libre des partisans.

12. 12 septembre 1944. Sous la pression anglaise, le roi Pierre II, qui avait confié en mai la responsabilité de son gouvernement au Dr Ivan Šubašić, ancien ban de Croatie, appelle par la 
radio ses sujets à se rallier à l'armée de libération nationale et reconnaît Tito comme chef de toutes les forces combattantes de Yougoslavie.

13. Il pense à la rencontre entre le maréchal Tito et le maréchal britannique Harold Alexander, commandant en chef des armées alliées en Italie ; cette rencontre avait pour sujet la question de Trieste.

14. Par «nos Turcs », Obrad comprend la population musulmane vivant dans ce coin des Balkans. 15. Magyars : Hongrois.

16. Bačka, région de la Voïvodine, où, en hiver 1942, la population juive, serbe et tzigane, fut massacrée suite aux ordres de Miklos Horthy.

17. Kajkavien, le dialecte parlé au nord de Zagreb. Le serbo-croate comprend trois dialectes que l'on désigne d'après les trois formes respectives du pronom relatif-interrogatif « que » (šta ou što, kaj et ča) : le štokavien (štokavski), le kajkavien (kajkavski) et le čakavien (čakavski). Le dialecte kajkavien est à la base de la langue slovène.

18. Le dialecte štokavien est parlé dans le reste du pays (Slavonie, Centre, Lika et une partie de Dalmatie) et il est la base de la langue littéraire croate.

19. En anglais dans le texte original. «Romains sauvages, cœurs durs, blocs de pierre, pire que choses insensibles, n'avez-vous pas connu Pompée? Que de fois, perchés aux murs et aux créneaux, aux tours et aux fenêtres, et jusque tout en haut des cheminées, vos petits dans vos bras, vous attendiez un jour entier, long comme une vie, pour voir le grand Pompée passer les rues de Rome " : Shakespeare W., Jules César, acte I, scène 1. Nous utilisons la traduction anglaise d'Edmond Fleg : Shakespeare W., Euvres complètes, t. II, Gallimard, Paris, 1959, p. 557.

20. Petar Nikolajević Moler, un des voïvodes de la première insurrection serbe, assassiné à Belgrade par le Vizir.Il a exigé de Miloš le partage du pouvoir et a proposé la tétrarchie où Miloš, lui-même et deux de ses collaborateurs, le voïvode Pavle Cukić et l'évêque Milentije Nikšić, auraient chacun trois districts à gouverner. Miloš, ne voulant pas partager le pouvoir, s'est occupé de l'ennemi intérieur à sa façon. Ils ont tous été assassinés.

21. Le voïvode Radič Petrović a été l'un des grands chefs de la première insurrection serbe.

22. Philiki Hétaïrie ou Philiki Hétairia (" société des amis ») est une organisation grecque de la fin du XVIII ${ }^{\mathrm{e}}$-début du XIX ${ }^{\mathrm{e}}$ siècle qui avait pour but de libérer les pays chrétiens du joug ottoman.

23. La Grèce.

24. En anglais dans le texte original. "Amis, Romains, concitoyens, prêtez-moi vos oreilles.» : Shakespeare W., Op. cit., acte III, scène 3, p. 587.

25. «Bravo », « bien joué » en turc.

\section{AUTEUR}

\section{NATAŠA DŽIGURSKI-DUBAJIĆ}

Lectrice de serbo-croate à l'Université Charles-de-Gaulle Lille3 (Villeneuve d'Ascq, France) 